\title{
GEOMETRÍA, ESQUEMAS E IDEALIZACIÓN: UNA MÓDICA DEFENSA DE LA FILOSOFÍA DE LA GEOMETRÍA DE KANT*
}

\author{
Álvaro J. Peláez Cedrés \\ apelaez@correo.cua.uam.mx \\ Universidad Autónoma Metropolitana-Cuajimalpa
}

\begin{abstract}
"¿Y sabías que, no obstante que utilizan las formas visibles y razonan sobre ellas, no están pensando en estas sino en las formas ideales a las cuales se asemejan; no en las figuras que dibujan, sino en el cuadrado y en el diámetro absolutos... (que) ellos en realidad están tratando de contemplar las cosas en sí mismas, que sólo pueden verse con los ojos de la mente?" (Platón, República).
\end{abstract}

\section{Resumen}

En este trabajo, defenderé la importancia de los esquemas trascendentales para intentar entender la clase de cosas que Kant tenía en mente cuando hablaba de representaciones u objetos geométricos. Argumentaré a favor de la idea de que, en general, los esquemas trascendentales constituyen un conjunto de reglas que permiten transformar una figura en otra, así como la representación de las propiedades que permanecen invariantes a través de las mismas, constituyendo los objetos genuinos de cognición en un juicio. Con ello, señalaré no solo las relaciones que, según mi opinión, tiene la filosofía kantiana de la geometría con la geometría de su época, sino también cómo la misma puede ser usada para entender los desarrollos posteriores de esta disciplina.

Palabras ClaVe: Kant, geometría proyectiva, esquemas, invariantes.

\section{Abstract}

In this work, I shall defend the importance of transcendental schemata for understanding the kind of things that Kant had in mind when he talked about geometric objects or representations. I will argue for the idea that in general, the transcendental schemata are a set of rules that allow the transformation of one figure into another, and also the representation of the properties that remain 
invariant under those rules, which are the genuine objects of cognition in a judgment. Likewise, I will show not only the relations that, in my opinion, the Kantian philosophy has with the geometry of his time, but also how such a philosophy can be used for understanding the late development of that discipline.

KeYwords: Kant, projective geometry, invariants, schemas.

\section{Introducción}

$\overline{\mathrm{R}}$ Desde el trabajo fundamental de los filósofos de la geometría de comienzos del siglo XX, Russell, Carnap, Schlick y Reichenbach, la teoría crítica de la geometría de Kant no ha resultado muy atractiva. Después del trabajo de dichos filósofos, y de la obra de Riemann, Hilbert y Einstein, en quienes aquellos se inspiraron, la concepción de Kant fue vista como un mero pintoresquismo. Su imagen de la geometría en cuanto basada en nuestra intuición del espacio parece abiertamente equivocada, y hubo una tendencia consecuente a ver la Estética Trascendental como un episodio desafortunado que uno ha de ignorar con vistas a una comprensión cabal de la Analítica.

La opinión estándar contra Kant sostuvo lo siguiente ${ }^{1}$ : Kant se equivocó al no reconocer la distinción crucial entre geometría pura y aplicada. La geometría pura es el estudio de las relaciones lógicas o formales entre proposiciones en un sistema axiomático particular, un sistema axiomático para la geometría euclidiana, por ejemplo. Como tal es a priori y cierto (tan cierto como lo es la lógica), pero no envuelve apelación alguna a la intuición espacial o alguna otra clase de experiencia. La geometría aplicada, por otro lado, concierne a la verdad o falsedad de un sistema de axiomas bajo una interpretación particular en el mundo real. Y, en conexión con esto, importa poco si nuestros axiomas son interpretados en el mundo físico -en términos de rayos de luz, barras rígidas o cualquier otra cosa, o en el reino psicológico- en términos de apariencias u otras entidades fenomenológicas. En cualquier caso, la verdad de cualquier sistema axiomático particular no puede ser a priori sino una cuestión de investigación empírica, en física o psicología. Esta actitud moderna quedó expresada en el famoso dictum de Einstein de que: "En la medida en que las leyes de las matemáticas refieren a la realidad, no son ciertas; y en la medida en que son ciertas, no refieren a la realidad" (Einstein 1922, p. 28). Desde este punto de vista, entonces, Kant malentiende las cosas desde el principio y por lo tanto sus enseñanzas son irremediablemente confusas.

1 Esta postura se encuentra en Schlick (1913), (1915) y (1922) y Reichenbach (1920) y (1958). La postura de Carnap en su (1922) es más compleja, pues al tiempo que separa la geometría matemática de la geometría física, reconoce la existencia de lo que llama, siguiendo a Kant, "espacio intuitivo". 
Los filósofos que así respondieron a la elucidación kantiana del conocimiento geométrico, lo hicieron principalmente inspirados en los cambios revolucionarios ocurridos en las matemáticas del siglo XIX, el surgimiento de las geometrías no-euclidianas, la formalización de la geometría euclidiana por parte de Hilbert y la rigorización del análisis, constituyendo el blanco de las críticas, la noción kantiana de intuición pura.

En los últimos años se han propuesto lecturas interesantes acerca del papel de la intuición en la teoría de la geometría de Kant. Jaakko Hintikka ${ }^{2}$, inspirado en el trabajo seminal de Evert Beth, sostiene que el papel primario de la intuición kantiana es formal o inferencial: sirve, a través del recurso a las construcciones, para generar términos singulares en el contexto del razonamiento matemático, en inferencias tales como la que nos representaríamos hoy mediante la instanciación existencial. De esta forma, el rasgo fundamental que distingue las intuiciones kantianas de las representaciones conceptuales es su singularidad como opuesta a la generalidad de los conceptos. Asimismo, Hintikka sugiere que el recurso a las construcciones, con la consiguiente necesidad de la intuición como medio en el cual llevar a cabo las mismas, es algo que Kant toma prestado de la geometría, en particular de la geometría sintética.

Estoy esencialmente de acuerdo con la idea de Hintikka de que Kant estaba intentando avenirse filosóficamente con el método sintético en geometría, con su apelación a las construcciones como recurso esencial de la producción de los objetos geométricos, es más, estoy de acuerdo con él aun en su afirmación de que Kant extiende dicho método a la producción de todo el conocimiento sintético. No obstante, pienso que Hintikka, al restringir su atención a la versión más antigua de este método geométrico, presente en Euclides, pasando por alto varios de los desarrollos que dicho método había sufrido en las matemáticas del siglo XVII, de la mano de eminentes geómetras como Desargues y Pascal, no hace completa justicia a la manera en que Kant mismo se estaba inspirando en el método geométrico en cuestión. Pienso en el impulso que tanto Desargues como Pascal le dieron al estudio de las secciones cónicas, y sobre todo a su preocupación por la cuestión de las propiedades compartidas por las diferentes secciones de una proyección, así como al papel de los elementos imaginarios en sus sistemas geométricos.

Desde mi punto de vista, Kant, como otros filósofos antes que él ${ }^{3}$, tomó muy en serio las preocupaciones de los geómetras contemporáneos, que ya habían criticado al método antiguo por su dependencia de las figuras particulares y por la imposibilidad de subsumir una diversidad aparentemente diferente de ellas bajo un concepto simple, y trató de combinar la idea de construcción con la de una representación emergente que expresara las propiedades compartidas por un cierto dominio de objetos. Esta representación, que no está restringida solo a las geométricas, sino a todo el dominio de la cognición sintética, es lo que, en mi opinión, Kant llama esquema de un concepto. 
En este trabajo defenderé la importancia de los esquemas trascendentales para entender la clase de cosas que Kant tenía en mente cuando hablaba de representaciones u objetos geométricos. Argumentaré a favor de la idea de que, en general, los esquemas trascendentales constituyen representaciones de las propiedades invariantes de una variedad de objetos, constituyendo los objetos genuinos de cognición en un juicio. Con ello, señalaré no solo las relaciones que, según mi opinión, tiene la filosofía kantiana de la geometría con la geometría de su época, sino también cómo la misma puede ser usada para entender los desarrollos posteriores de esta disciplina.

\section{El contexto geométrico del siglo XVII}

Como he dicho antes, estoy fundamentalmente de acuerdo con Hintikka en que Kant se inspiró en el método de la geometría sintética no solo para su filosofía de la geometría, sino también para la articulación de parte de su sistema epistemológico. Como bien apunta Hintikka, la clave de este método, el cual se remonta a los griegos y está explícito en Euclides, se encuentra en el concepto de construcción. Su aplicación consiste en tratar de producir un resultado mediante la efectuación real de construcciones, y lo que es más importante, que dichas construcciones proceden desde elementos simples a partir de un conjunto fijo de reglas. Como Hintikka dice correctamente, una parte importante de la prueba de un teorema del sistema de Euclides, la $\varepsilon \dot{x} \theta \dot{\varepsilon} \sigma ı \varsigma$, procede mediante la construcción de la figura enunciada en la primera parte del teorema, la $\pi \varrho o ́ \tau \alpha \sigma ı \varsigma$. Hasta aquí no hay problemas. Los problemas surgen cuando los geómetras se percataron de que esta dependencia de las construcciones literales, es decir, de los particulares que se construyen con regla y compás en el encerado, conducía a dos hechos inaceptables prima facie, a saber, que aquellas cosas que manifestaban características visibles diferentes no pudieran ser subsumidas bajo un concepto simple; y la carencia de unidad en los principios constructivos de la geometría.

Esta situación comenzó a revertirse con el trabajo de Descartes, quien planteó de una manera explícita el principio de que todas las expresiones particulares del pensamiento han de presentar un orden y conexión definida. No es el contenido de un pensamiento dado lo que determina su valor cognoscitivo, sino la necesidad mediante la cual se deduce desde primeros principios en una secuencia ininterrumpida. La primera regla de todo el conocimiento racional es, entonces, que las cogniciones sean ordenadas formando una serie autocontenida dentro de la cual no hay transiciones nomediadas. Ningún miembro puede ser introducido como un elemento enteramente nuevo, sino que cada uno ha de surgir paso a paso desde miembros anteriores de acuerdo con una regla.

Este pensamiento fundamental de Descartes demandó y condicionó una nueva concepción de la geometría. El conocimiento geométrico en sentido estricto no se encuentra donde los particulares son estudiados como objetos aislados, sino solo donde la totalidad de esos objetos puede ser constructivamente generada de acuerdo a un proceso dado. La geometría sintética antigua viola este postulado, porque su objeto es 
la figura espacial aislada, cuyas propiedades se aprehenden en la intuición sensible inmediata, pero cuya conexión sistemática con otras figuras nunca puede ser representada completamente. En este punto, de acuerdo con Descartes, la geometría solo puede ser completada a través de su determinación por medios aritméticos. El fin del método filosófico consiste en concebir a todos los objetos con la misma conexión sistemática que poseen los objetos aritméticos.

Ahora bien, en el desarrollo posterior, la geometría nunca se iba a apartar de este principio cartesiano fundamental. Sin embargo, sí lo haría en relación con otro punto de fundamental importancia, a saber, el rechazo a la algebrización de la geometría y la consiguiente restauración del papel de la intuición en las cuestiones propiamente geométricas. Ya Leibniz había criticado a la geometría analítica por introducir un elemento arbitrario en la determinación de las figuras espaciales, a saber, los diferentes sistemas de coordenadas y sus diferentes ecuaciones. Desde el punto de vista de la que luego se llamaría, en el siglo XIX, geometría de la posición o proyectiva, y que en el siglo XVII no se distinguía nominalmente de la geometría euclidiana, lugar desde donde se operó la divergencia con la geometría analítica, no es cuando limitamos la intuición y buscamos reemplazarla por meras operaciones de cálculo que obtenemos las verdaderas construcciones lógica y estrictamente deductivas de la ciencia del espacio, sino cuando colocamos a la intuición en su completo alcance e independencia.

El problema fundamental, ya referido en la introducción, al cual se enfrentaron los geómetras del siglo XVII, problema que, por otra parte, ya había sido planteado por Alberti, fue: ¿qué propiedades geométricas tienen en común dos secciones de la misma proyección de una figura actual? El primero que dio una respuesta a esta pregunta en términos sistemáticos fue Girard Desargues (1591-1661).

Un aspecto importante de su sistema geométrico fue la introducción de elementos imaginarios en el plano euclidiano. Esto fue posible por su tratamiento de las líneas paralelas, las cuales concibió como un caso de líneas que se intersectan en un punto en común, el punto de intersección, el cual era trasladado al infinito. A su vez, esto lo condujo a uno de sus resultados más conocidos, el ahora llamado "teorema de Desargues". A este deben agregarse, entre otros resultados, el concepto de involución (que había sido introducido por Papo de Alejandría), y el de conjunto armónico de puntos.

En mi opinión, esta cuestión acerca de la introducción de elementos imaginarios en el sistema geométrico no tiene únicamente una importancia fundamental desde el punto de vista de la historia de las matemáticas, sino también desde un punto de vista epistemológico. Dicha importancia radica en que revolucionó el modo de entender la cuestión de la naturaleza de las representaciones geométricas. Por ejemplo, si consideramos un círculo y una línea recta que la interseca, podemos transformar este sistema geométrico mediante desplazamientos continuos de una manera tal que finalmente la línea recta queda completamente fuera del círculo, de modo que las intersecciones y las direcciones de los radios que corresponden a ellas han de ser expresadas por valores imaginarios. La coordinación de la figura deducida con la original ya no 
conecta elementos que son actualmente presentes y observables, sino elementos meramente intelectuales; se ha resuelto en una correlación ideal. En esta visión, el objeto real de la investigación geométrica no es la forma individual en su existencia sensorial, sino las diversas clases de dependencia que pueden subsistir entre las formas.

\section{Idealizaciones y esquemas trascendentales}

De acuerdo con la distinción entre los usos de la razón que Kant establece en la Doctrina transcendental del método, el conocimiento filosófico es conocimiento racional derivado de conceptos, mientras que el conocimiento matemático lo es por construcción de conceptos. Aquí ya parece haber evidencia a favor del énfasis de Hintikka en el papel que las construcciones tendrán en la concepción kantiana. Sin embargo, veamos un poco más de cerca qué nos dice Kant acerca de esas construcciones. Dice: "Para construir un concepto hace falta, pues, una intuición no empírica que, consiguientemente, es, en cuanto intuición, un objeto singular, a pesar de lo cual, en cuanto construcción de un concepto (representación universal), tiene que expresar en su representación una validez universal en relación con todas las posibles intuiciones pertenecientes al mismo concepto" (A714-B742). De acuerdo con un presupuesto fundamental de la filosofía kantiana, a todo concepto debe corresponderle una intuición, la cual, por supuesto, viene suministrada por la sensibilidad. En el caso de las matemáticas, que procede constructivamente en relación con todos sus conceptos, sus intuiciones han de proveerse a priori. Dado que la misma constituye el objeto que otorga significado al concepto y así el objeto al que este refiere, esta no puede ser una mera representación particular, sino, como dice Kant, una que exprese las propiedades compartidas por todas las intuiciones pertenecientes al mismo concepto. Al hablar de propiedades compartidas, aclara Kant unas líneas más abajo, no nos referimos a las propiedades prescindibles de una cierta clase de objetos, como serían, en un triángulo, la magnitud de los lados y de los ángulos, sino a las propiedades universales y necesarias de los mismos. Esta intuición, según él, "apunta siempre al simple acto de construir el concepto" (A714-B742), esto es, constituye una representación cuyo fin es la expresión de un concepto. Por ello no puede ser meramente particular.

Esta representación intuitiva pero determinada conceptualmente, la cual constituye el objeto del concepto, y es construida, en palabras de Kant "sin tomar el modelo de una experiencia" (ídem), es el esquema del concepto. Así lo expresa Kant en el mismo apartado: "Por ello, así como este singular se halla determinado por ciertas condiciones universales de la construcción, así también el objeto del concepto, al que dicho singular corresponde como su mero esquema, tiene que concebirse como universalmente determinado" (ídem).

Desde el punto de vista de los desarrollos en geometría proyectiva que comentamos en nuestro apartado anterior, esto tiene pleno sentido. En tanto objetos concretos, las figuras geométricas son diferentes, pero a nivel abstracto son las mismas. Por ejemplo, el cubo y el octaedro son objetos intuitivamente diferentes. No obstante, sus 
respectivos grupos de automorfismos tienen la misma estructura algebraica, por lo que decimos que uno es el dual del otro. Y lo mismo ocurre con el dodecaedro y el icosaedro. Constituyen, al igual que los puntos al infinito que mencionamos antes, objetos imaginarios, ideales, constructos abstractos que expresan propiedades universales determinadas conceptualmente.

Por ello, también es natural que Kant considere el papel de la imaginación en la construcción de los objetos matemáticos. En las Observaciones generales sobre la estética trascendental, Kant se pregunta acerca de las proposiciones de la geometría: “¿de dónde sacamos semejantes proposiciones y en qué se apoya nuestro entendimiento para llegar a tales verdades absolutamente necesarias y universalmente válidas?" (B64-A47). Y la respuesta es que un conocimiento de ese tipo solo podría obtenerse de dos fuentes, a saber, intuiciones o conceptos. Dado que ambos están dados a priori o a posteriori, debemos considerar cada una de estas opciones. Es claro que, si como Kant parece creer (y nadie en el siglo XVIII creería lo contrario ${ }^{4}$ ), la geometría es una disciplina constituida por verdades universales y necesarias, no podría derivar sus proposiciones de conceptos empíricos y de sus intuiciones correspondientes, pues dicha cosa convertiría a la geometría en una disciplina irremediablemente empírica. Solo quedan las opciones de que el conocimiento geométrico se derive o bien de conceptos o bien de intuiciones a priori. Lo primero es desechado apelando a la definición de analiticidad y a la imposibilidad de explicar un juicio de la geometría en base a dicha idea. Entonces, la única opción que queda es considerar que las proposiciones de la geometría se derivan de intuiciones puras. Pero aquí Kant plantea una distinción, dice: "Pero, ¿de qué clase de intuición pura se trata: a priori o empírica?" (A48-B65). En mi opinión, una intuición pura empírica sería una representación inmediata de un momento particular del tiempo o un espacio particular no actual, por ejemplo, la representación que puedo tener en el momento en que escribo este trabajo de las turbias aguas del Río de la Plata. Se trata de una representación independiente de la experiencia del caso, pero no independiente de toda experiencia. $\mathrm{Y}$ es posible por la intervención de la facultad de imaginación, aunque de una imaginación puramente reproductiva. Asimismo, se trataría de una representación que no es universalmente válida, sino de una que tiene una validez puramente subjetiva. Por otro lado, una intuición pura a priori sería una representación que surge como una necesidad de la forma pura de la sensibilidad, independientemente de los caracteres sensibles pasados o actuales, aunque no completamente independiente de toda clase de representación sensible. La facultad que interviene en esta construcción es también la imaginación, aunque en este caso su naturaleza es esencialmente productiva. Dice Kant: "En esta síntesis sucesiva de la imaginación productiva se basan, para producir

4 En el siglo XIX en cambio, el surgimiento de las geometrías no-euclidianas hizo pensar a algunos que la geometría podría ser empírica. Lobachevsky cotejó observaciones astronómicas para determinar la constante de su geometría. Usó un triángulo formado por Sirio, Rigel y la estrella 29, pero el defecto era demasiado pequeño para ser significativo. Sobre este punto, véase Torretti (1978) pp. 63-64. 
las figuras, las matemáticas de la extensión (geometría) con sus axiomas” (B204). Es decir, la geometría procede desde ciertos elementos básicos o primitivos, regida por sus axiomas, para producir objetos imaginarios, ideales, representaciones que aprehenden las propiedades universales de una clase de figuras. Construye, para volver nuevamente a la terminología específica kantiana, esquemas.

Permítaseme para terminar mi argumento acerca del carácter no particular de las representaciones asociadas con las construcciones en geometría, considerar algunas de las observaciones de Kant sobre la naturaleza de dichos esquemas. En la sección sobre el esquematismo de los conceptos puros del entendimiento de la Crítica de la razón pura $(C R P)$, Kant parte del reconocimiento de que los esquemas son producto de la imaginación, pero enfatiza de inmediato que, dado que la imaginación tiene aquí la función de sintetizar una multiplicidad de intuiciones, su resultado no puede ser una intuición particular. Así, un esquema ha de ser distinguido cuidadosamente de una imagen, la cual en sentido estricto lo es siempre de un objeto particular. En el ejemplo que proporciona Kant, los cinco puntos ... constituyen una imagen, una representación sensible del numero cinco, lo que no quiere decir que constituya, desde su punto de vista, la intuición relacionada con el concepto de cinco. A favor de ello, alega Kant que dicha representación particular nunca podría ser comparada con el concepto. Es decir, nunca podría constituir el objeto genuino de referencia del concepto. Y ejemplifica una vez más con un caso tomado de la geometría: "Ninguna imagen de un triángulo se adecuaría jamás al concepto de triángulo en general. En efecto, la imagen no alcanzaría la universalidad conceptual que hace que el concepto sea válido en relación con todos los triángulos, sean rectángulos, oblicuángulos, etc., sino que siempre estaría limitada a una sola parte de esa esfera. El esquema del triángulo no puede existir más que en el pensamiento, y significa una regla de síntesis de la imaginación respecto de figuras puras en el espacio" (A141-B180).

Desde mi punto de vista, aunque Kant se muestre ciertamente pesimista en torno a la cuestión de qué sean esos esquemas y cuál sea la clave de su comprensión, el enunciado final del párrafo citado es profundamente significativo. En mi opinión, no solo explica algunos de los avances, aunque incipientes, en el desarrollo de la geometría de su época, sino que dicha explicación puede aplicarse a desarrollos posteriores en la misma línea. Trataré este último aspecto en la siguiente sección.

\section{Conclusiones. Esquemas, idealizaciones e invariantes geométricos}

Permítaseme retomar brevemente la historia que conté en la sección segunda. Los siguientes nombres de importancia en el desarrollo de la geometría de la posición o proyectiva fueron los de Poncelet, Gergonne, Grassman y von Staudt; el problema al que se enfrentaron, el mismo que había preocupado a Desarges y Pascal, la justificación de los elementos imaginarios. Por mor de la brevedad solo consideraré al primero y al último de dichos nombres. 
Poncelet hizo esfuerzos importantes por justificar la introducción de los elementos imaginarios en los sistemas geométricos, pero problematizó al mismo tiempo la dependencia de la geometría sintética de los diagramas explícitamente trazados. Reconociendo la superioridad de los métodos algebraicos en el tratamiento de los problemas geométricos, pero interesado al mismo tiempo por retornar al ideal de lo geométrico como la teoría de lo propiamente espacial, se preguntó si la geometría sintética no podría incorporar métodos tan potentes y efectivos como los del álgebra, embarcándose en una reinterpretación de los contenidos de la geometría.

El primer rasgo importante que Poncelet observó en el álgebra es que esta opera con signos abstractos. Dice al respecto: "El álgebra emplea signos abstractos, representa magnitudes absolutas mediante caracteres que no tienen valor en sí mismos, y que permiten a dichas magnitudes toda la indeterminación posible; por consiguiente, operan y razonan forzosamente tanto sobre signos de no-existencia como sobre cantidades absolutas y reales: $a$ y $b$, por ejemplo, representan dos cantidades cualesquiera, y es imposible en el curso del cálculo, recordar y reconocer cuál es el orden de sus magnitudes numéricas; a pesar de ello, somos llevados a razonar sobre las expresiones $a-b, \sqrt{a-b}$, etc., como si se tratara de cantidades siempre absolutas y reales. Los resultados deben, por consiguiente, participar de esta generalidad, y se extiende a todos los casos posibles, a todos los valores de las letras que se introducen; de esta manera, son estas formas extraordinarias, estos seres de razón, que parecen ser la posesión exclusiva del álgebra" (Poncelet 1822, pp. XX-XXI).

Poncelet agrega que todas las disciplinas que empleen este mismo tipo de signos abstractos estarán en situación de explotar las ventajas del análisis algebraico, y si no lo han hecho, como la geometría sintética, es porque se ha estado aferrado dogmáticamente al uso y significado de los diagramas. Sin embargo, no se trata finalmente de borrar la frontera entre geometría sintética y analítica, sino de reinterpretar el uso y la significación de los diagramas empleados en la primera ${ }^{5}$. El paso dado por Poncelet consiste en no tomar a un diagrama particular dado como el objeto de estudio de la geometría, sino antes bien como un signo complejo cuyos componentes pueden ser operados sin tomar en cuenta las particularidades que se siguen de sus caracteres visualizables, y de allí obtener propiedades generales de las figuras. Más específicamente, Poncelet argumentó que en los casos en los cuales una forma persiste, pero el objeto que la acompaña se desvanece, se deben postular nuevos elementos de acuerdo al principio general de persistencia de la forma, el cual podría parafrasearse de la siguiente manera: si suponemos que una figura dada cambia su posición al sufrir cada uno de sus puntos un movimiento continuo sin violar las condiciones que

5 Uno de los primeros que vieron la necesidad de reformular el sentido de lo que se entendía por "intuición” fue Jacob Steiner, quien sostuvo que el significado de la intuición no es la adherencia a una figura sensorialmente dada, sino que es la libre generación constructiva de figuras de acuerdo a un principio unitario. Los diferentes casos de figuras sensorialmente dadas no son, como en la geometría antigua, individualmente concebidos y estudiados, sino que todo el interés se concentra en la manera en la cual ellas proceden mutuamente unas de otras. 
inicialmente se sostienen entre ellos, las propiedades que se sostienen para la primera posición de la figura, se sostienen todavía en una forma generalizada para todas las figuras derivadas.

La fuerza y conclusividad de toda prueba geométrica descansa en los invariantes del sistema, no en lo que es peculiar a los miembros individuales como tales. El único postulado que está implicado puede ser formulado diciendo que es posible mantener la validez de ciertas relaciones, definidas de una vez y para siempre, a pesar del cambio en el contenido de los términos particulares. Comenzamos considerando a la figura en una conexión general, y no la analizamos al comienzo en sus partes individuales, sino que permitimos ciertos cambios dentro de una cierta esfera definida por las condiciones del sistema. Si esos cambios proceden continuamente desde un punto de partida definido, las propiedades sistemáticas que hemos descubierto en una figura serán transferibles a cada "fase" sucesiva, de modo que las determinaciones finales que se encuentran en un caso individual pueden ser progresivamente extendidas a todos los miembros sucesivos. Como Poncelet enfatiza, nunca son las meras propiedades particulares de una figura desde las cuales comienza el tratamiento proyectivo, sino desde las propiedades de un "género", donde "género" significa nada más que una conexión de condiciones mediante las cuales todo lo individual es ordenado. Todas las formas que pueden surgir una de otra en esta forma son consideradas como una unidad indivisible, son expresiones diferentes de uno y el mismo concepto. Obviamente, "pertenecer a un concepto" no significa aquí el que los particulares compartan ciertas semejanzas genéricas, sino la presuposición de un cierto principio de transformación que se mantiene idéntico.

Ahora bien, entre 1847 y 1860, el geómetra alemán G. K. C. von Staudt trajo una nueva perspectiva al problema del estatus de los imaginarios en geometría proyectiva. Antes que postular nuevas entidades a la manera de Poncelet, von Staudt optó por definirlos en términos de ideas euclideanas aceptadas, de modo que la geometría proyectiva debía ser vista como una extensión del viejo campo, mediante definiciones. No obstante, la originalidad de von Staudt no radica en este propósito de extensión de la geometría euclidiana, sino en su particular estrategia para obtener tal fin. Dado que no hay objetos euclidianos regulares que puedan sustituir los puntos complejos, von Staudt se vio forzado a tratar con un material inesperado. La solución fue seleccionar conceptos abstractos para suministrar el material faltante. Dice von Staudt: "Estamos familiarizados con casos donde las aseveraciones sobre conceptos o relaciones se hacen de acuerdo a algo como un objeto, como cuando decimos "El ser persona es poseído por Sócrates", antes que "Sócrates es una persona". Considérese "ser persona" como un concepto-objeto (Begriffsgegenstand) derivado del concepto "es una persona". Desde este punto de vista, considérese la aparentemente inocente afirmación: "La línea $L$ tiene una involución (definida por $x x^{\prime}=-10$ )". Una vez más, hemos convertido lo que es esencialmente una relación (" $x$ mapea a $x$ ' mediante la regla $x x^{\prime}=-10$ ") en el concepto-objeto "la involución en L definida por $x x^{\prime}=-10$ ". Recuérdese que Poncelet postuló nuevos puntos que correspondieran a los "puntos fijos" de tal relación... Pero, antes que seguir a Poncelet y ver a este proceso como de postulación, ¿por qué no simplemente definir los puntos imaginarios como los 
concepto-objetos denotados por frases como «la involución en L»?" (Citado en Wilson 1995, p. 126).

Aunque la mayoría de los textos de la época incluían secciones sobre "abstracción" que eran similares en este respecto a las ideas de von Staudt, nadie antes que él había intentado definir objetos concretos como puntos en términos de esas entidades abstractas. Mientras Poncelet creía que era imprescindible postular nuevos elementos en orden a obtener la "persistencia de la forma", von Staudt vio que las mismas formas persistentes, reconstituidas en el ropaje de los concepto-objetos, podrían servir como los elementos buscados.

Tal vez ya parezca obvio que estoy queriendo sugerir la familiaridad de estas nociones relacionadas a las propiedades permanentes de los objetos geométricos a través de ciertos cambios, con la noción kantiana de esquema. Como decíamos antes, Kant parece pensar que un esquema constituye al mismo tiempo, tanto la regla que permite conectar o derivar una figura desde otra, como una especie de representación asociada, abstracta, ideal, que captura las propiedades compartidas por esa colección de objetos.

En esta conexión, permítaseme agregar un episodio más en la historia de la geometría que puede verse a la luz del concepto kantiano de esquema. Se trata del que con justicia podría considerarse el paso decisivo hacia una formulación completa del concepto geométrico en tanto que estructura que persiste a través de los cambios en sus aplicaciones particulares, a saber, la introducción de la teoría de grupos ${ }^{6}$ en la geometría debida a F. Klein. En el concepto de grupo se obtiene un principio general de clasificación mediante el cual los diferentes tipos de geometrías pueden ser unificados bajo un punto de vista simple. Si planteamos la pregunta acerca de qué debemos considerar como una geometría, la respuesta es: aquellas propiedades que permanecen invariantes a través de ciertas transformaciones espaciales. Es decir, aquellas estructuras que persisten cuando variamos la posición absoluta de esta estructura en el espacio, cuando aumentamos o disminuimos proporcionalmente la magnitud absoluta de sus partes o cuando finalmente revertimos la ordenación de las partes individuales, como cuando sustituimos la figura original por otra que se relaciona con ella como con su imagen en un espejo.

Así, por ejemplo, la geometría euclideana es el estudio de las propiedades invariantes bajo el grupo de los así llamados movimientos rígidos, a saber, traslación, rotación y reflexión. La propiedad esencial preservada por este grupo de movimientos es la distancia, es decir, la característica de la isometría ${ }^{7}$.

6 Un grupo se define como un conjunto $\mathrm{G}$ no vacío junto con una operación binaria (*), tal que: para cualesquiera $g_{1}$ y $g_{2}$ de $G, g_{1} * g_{2}$ es un elemento de $G$; la operación es asociativa; el grupo contiene el elemento de identidad; y para cada elemento existe un inverso.

7 Una transformación $f$ es una isometría de $\mathrm{A}$ sobre $\mathrm{B}$ si preserva las distancias. Para cualesquiera dos puntos $\mathrm{P}_{1}, \mathrm{P}_{2}$ de $\mathrm{A}$, la distancia desde $\mathrm{P}_{1}$ a $\mathrm{P}_{2}$ es igual a la distancia desde $f\left(\mathrm{P}_{1}\right)$ a $f\left(\mathrm{P}_{2}\right)$. 
De este modo, los verdaderos objetos de consideración e importancia para el geómetra no son los particulares, sino las propiedades que, a través de ciertos grupos de transformaciones, permanecen invariantes. El esquema, para usar de nuevo la terminología kantiana, equivale en este punto al conjunto de reglas que permiten transformar una figura en otra. Pero no solo eso, el esquema sería la representación intuitiva que captura las propiedades esenciales que emergen del acto de transformación.

Klein mismo distinguió entre la que llamó una intuición ingenua y una intuición refinada ${ }^{8}$. La primera está constituida por la asociación de un concepto con una representación sensible; la segunda, en cambio, es aquella en la que los axiomas constituyen e idealizan las intuiciones en la forma de representaciones universales.

Así, parece que aunque los filósofos han encontrado a las nociones kantianas de intuición pura, imaginación, esquemas y otras relacionadas, como irremediablemente oscuras, Kant no parece haber estado completamente equivocado al utilizar dichas nociones para elucidar la práctica geométrica. Como he intentado mostrar, su elucidación hace justicia no solo a la geometría tal como se practicaba en su tiempo, sino a sus desarrollos subsiguientes.

\section{Referencias bibliográficas}

Carnap, R. (1922), “Der Raum. Ein Beitrag zur Wissenschaftslehre”, en Kantstudien Ergänzungshefte, 56. Traducción inédita al inglés de Michael Friedman y Peter Heath como Space: A contribution to the theory of science.

Einstein, A. (1922), Sidelights on Relativity. Londres: Dodo Press.

Hintikka, J. (1967), "Kant on the Mathematical Method", en The Monist. 51: 352375.

(1969), “On Kant's Notion of Intuition”, en T. Penelhum y J. MacIntosh (eds.), Kant's First Critique. California: Wadsword.

Hobbes, T. (1987), “Sobre el cuerpo” en Hobbes: Antología. Barcelona: Península.

Klein, F. (1911), "On the Mathematical Character of Space-Intuition and the Relation of Pure Mathematics to the Applied Sciences", en Ewald, W. (comp.), From Kant to Hilbert: A Source Book in the Foundations of Mathematics, Oxford: Clarendon Press (1996).

Nagel, E. (1979), "The Formation of Modern Conceptions of Formal Logic in the Development of Geometry", en Teleology Revisited and Other Essays in the Philosophy and History of Science. Nueva York: Columbia U. Press.

Parsons, Ch. (1969), "Kant's Philosophy of Arithmetic", en Morgenbesser, S., Suppes, P., y M. White, (eds.), Philosophy, Science and Method: Essays in Honor of 
Ernest Nagel, St. Martin Press, reimpreso en Posy, C. (ed.), Kant's Philosophy of Mathematics. Modern Essays. Holanda: Kluwer, 1992.

Poncelet, J. V. (1822), Traité de proprietés projectives des figures. Paris: Bachelier. Reichenbach, H. (1920), Relativitätstheorie und Erkenntnis a priori. Trad. inglesa, The Theory of Relativity and a priori Knowledge. Los Angeles, 1965.

(1958), The Philosophy of Space and Time. New York: Dover.

Schlick, M. (1913), “Is There Intuitive Knowledge?”, en Philosophical Papers, vol.1 1909-1922. Holanda: Reidel, 1979.

(1915), "The Philosophical Significance of the Principle of Relativity", en Philosophical Papers, vol.1 1909-1922. Holanda: Reidel, 1979.

(1922), "Space and Time in Contemporary Physics" en Philosophical Papers, vol.1 1909-1922. Holanda: Reidel, 1979.

Spinoza, B. (1989), Tratado de la reforma del entendimiento. Madrid: Tecnos.

Torretti, R. (1978), Philosophy of Geometry from Riemann to Poincaré. Dordrecht: Reidel.

Wilson, M. (1995), "Frege: The Royal Road from Geometry", en Demopoulos, W. (ed.), Frege `s Philosophy of Mathematics. Cambridge: Harvard University Press. 